\title{
Tres proyecciones de la esfera al cilindro
}

\author{
Vinicio Antonio Gómez Gutiérrez \\ Facultad de Ciencias, Universidad Nacional Autónoma de México \\ e-mail: vgomez@ciencias.unam.mx
}

\begin{abstract}
Resumen
En este artículo se estudian tres transformaciones de la esfera al plano. Se trata de abordar el problema de hacer un mapa de una superficie esférica que tenga la característica de que los ángulos medidos en el mapa coincidan con los ángulos medidos en la superficie terrestre. Se muestra que ni la proyección central ni la proyección ortogonal al eje vertical tienen la propiedad deseada. Se da una deducción de la transformación de Mercator, la cual, por añadidura, nos muestra una aplicación de la integral de la secante.
\end{abstract}

\section{Introducción}

El problema de representar adecuadamente la superficie terrestre en un mapa fue aumentando su importancia a medida que los navegantes pudieron recorrer distancias cada vez más grandes, pues a mayor escala la influencia de la curvatura de la superficie terrestre es mayor. Esta influencia se traduce en que no se puede realizar un mapa en el cual distancias, áreas y ángulos queden representados adecuadamente. En particular, si los ángulos que se miden en un mapa no coinciden con los ángulos que forman las curvas correspondientes en la superficie terrestre, dicho mapa no podrá ser utilizado por un navegante para dirigir su barco en la dirección correcta. Esto podría tener consecuencias fatales. La transformación de Mercator pudo resolver esta necesidad. Sin embargo, en los libros clásicos de Cálculo Diferencial e Integral, o incluso de Geometría Diferencial, no se suele estudiar esta proyección. Algunos artículos han abordado la relación de la transformación de Mercator con la integral de la secante desde un punto de vista histórico (véase en especial [7, 1]). Un artículo más reciente [4] presenta una propuesta de implementación en la enseñanza de la integral. En opinión del que escribe estas líneas, muestra adecuadamente cómo calcular la transformación de Mercator, sin embargo, hay que argumentar más por qué esa transformación cumple las propiedades requeridas. En este artículo me propongo complementar los trabajos mencionados.

\section{Transformaciones Conformes}

Para tener un punto de partida común con el lector, empezaremos definiendo algunos conceptos, siguiendo a Palmas y a Reyes [6].

Definición 2.1 Un conjunto $S \subset \mathbb{R}^{3}$ es una superficie diferenciable si para cada punto $p \in S$ existe una vecindad $U$ de $p$ en $S$ y un difeomorfismo $\phi: \Omega \rightarrow U$ de una región $\Omega \subset \mathbb{R}^{2}$ en $U$. 
Un difeomorfismo es una función diferenciable con inversa diferenciable. Para saber más sobre este concepto recomendamos el libro de Guillemin y Pollack [3].

Definición 2.2 Sean $S_{1}$ y $S_{2} \subset \mathbb{R}^{3}$ dos superficies diferenciables. Una transformación $f: S_{1} \rightarrow S_{2}$ es una aplicación conforme si y sólo si $f$ es un difeomorfismo y existe una función $\lambda: S_{1} \rightarrow \mathbb{R}$ positiva y diferenciable tal que

$$
<d f_{p}\left(v_{1}\right), d f_{p}\left(v_{2}\right)>_{f(p)}=\lambda(p)<v_{1}, v_{2}>_{p}
$$

para todo punto $p \in S_{1}$ y cualesquiera vectores $v_{1}$, $v_{2}$ tangentes a $S_{1}$ en $p$.

En esta definición, $<,>_{p}$ denota la métrica Riemmaniana que nos da un producto escalar en el plano tangente a la superficie $S_{1}$ en el punto $p$. Análogamente $<,>_{f(p)}$ denota a la métrica Riemanniana que nos da un producto escalar en el plano tangente a $S_{2}$ en el punto $f(p)$. Sin embargo, para nuestros propósitos será suficiente el producto escalar inducido en el plano tangente por el producto escalar usual de $\mathbb{R}^{3}$.

Vamos a tratar de explicar con otras palabras qué significa que una transformación $f: S_{1} \rightarrow S_{2}$ sea conforme. Una primera aproximación es decir que $f$ preserva los ángulos entre las curvas. Para ser más precisos:

Sea

$$
\begin{gathered}
\alpha_{1}: \mathbb{R} \rightarrow S_{1} \\
t \mapsto\left(x_{1}(t), y_{1}(t), z_{1}(t)\right)
\end{gathered}
$$

una curva que representa el movimiento de un punto $\left(x_{1}, y_{1}, z_{1}\right)$ en la superficie $S_{1}$ a lo largo del tiempo $t$.

Sea

$$
\begin{gathered}
\alpha_{2}: \mathbb{R} \rightarrow S_{1} \\
t \mapsto\left(x_{2}(t), y_{2}(t), z_{2}(t)\right)
\end{gathered}
$$

una curva que representa el movimiento de un punto $\left(x_{2}, y_{2}, z_{2}\right)$ en la superficie $S_{1}$ a lo largo del tiempo $t$.

Supongamos que en el instante $t=t_{0}$ las dos curvas pasan por un mismo punto $P$. Esto significa que

$$
P=\left(x_{1}\left(t_{0}\right), y_{1}\left(t_{0}\right), z_{1}\left(t_{0}\right)\right)=\left(x_{2}\left(t_{0}\right), y_{2}\left(t_{0}\right), z_{2}\left(t_{0}\right)\right)
$$


El ángulo entre las curvas $\alpha_{1}$ y $\alpha_{2}$ en el punto $P$ se define como el ángulo entre los vectores tangentes

$$
\alpha_{1}^{\prime}\left(t_{0}\right)=\left(\frac{d x_{1}}{d t}\left(t_{0}\right), \frac{d y_{1}}{d t}\left(t_{0}\right), \frac{d z_{1}}{d t}\left(t_{0}\right)\right) \quad y \quad \alpha_{2}^{\prime}\left(t_{0}\right)=\left(\frac{d x_{2}}{d t}\left(t_{0}\right), \frac{d y_{2}}{d t}\left(t_{0}\right), \frac{d z_{2}}{d t}\left(t_{0}\right)\right)
$$

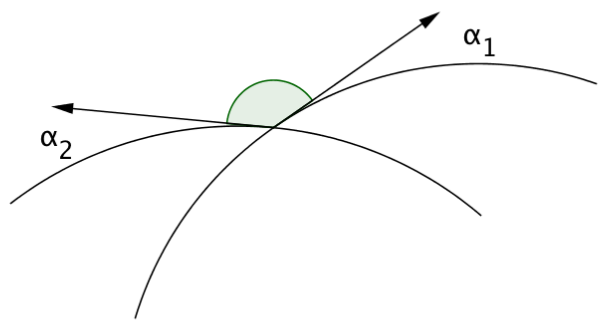

Figura 1: El ángulo entre dos curvas es el ángulo entre sus vectores tangentes

A cada una de las dos curvas

$$
\alpha_{i}: \mathbb{R} \rightarrow S_{1}
$$

le corresponde una curva

$$
\beta_{i}=f \circ \alpha_{i}: \mathbb{R} \rightarrow S_{2}
$$

Observemos el ángulo que forman las curvas $\beta_{1}$ y $\beta_{2}$ en el punto $Q=f(P)$. Si este ángulo resulta ser el mismo que el ángulo que formaban las curvas $\alpha_{1}$ y $\alpha_{2}$ en el punto $P$, y si esto pasa en todos los puntos de $S_{1}$ con las curvas correspondientes, diremos que $f$ preserva los ángulos.

En resumen: para verificar que una transformación tenga esta propiedad lo que debemos hacer es tomar un punto $P$ cualquiera en la superficie dominio, tomar dos curvas cualesquiera $\alpha_{1}$ y $\alpha_{2}$ que pasen por $P$ en un mismo instante $t_{0}$, medir el ángulo entre los vectores $\alpha_{1}^{\prime}\left(t_{0}\right)$ y $\alpha_{2}^{\prime}\left(t_{0}\right)$, luego tomar las curvas imagen $\beta_{1}$ y $\beta_{2}$, medir el ángulo entre los vectores $\beta_{1}^{\prime}\left(t_{0}\right) \mathrm{y}$ $\beta_{2}^{\prime}\left(t_{0}\right)$ y demostrar que ambos ángulos son iguales.

Apliquemos este procedimiento a una transformación que cumpla la definición de aplicación conforme: 


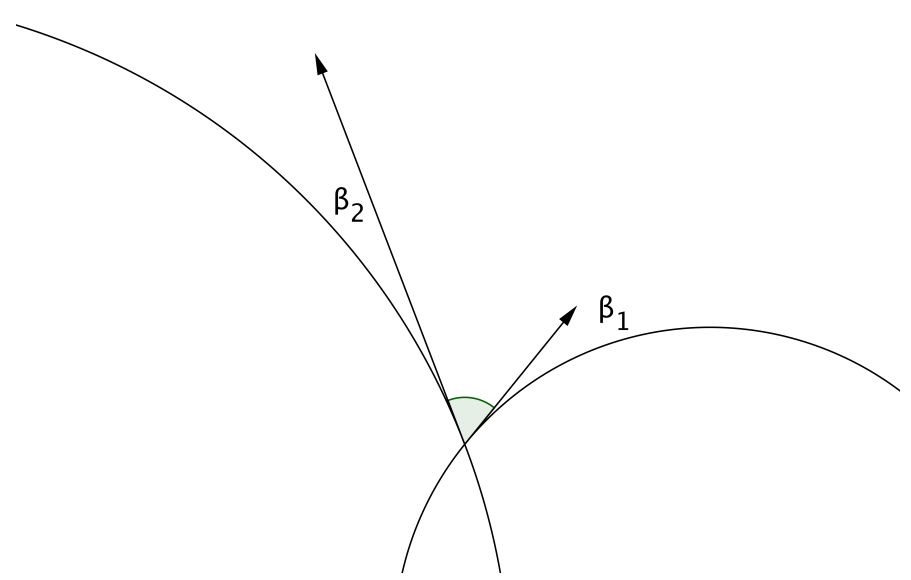

Figura 2: Las curvas imagen forman otro ángulo

Tomemos un punto $p \in S_{1}$. Luego tomemos dos vectores tangentes a $S_{1}$ en el punto $p$. Estos dos vectores pueden pensarse como vectores velocidad de curvas $\alpha_{1}(t)$ y $\alpha_{2}(t)$. Es decir, suponemos que:

$$
\begin{gathered}
p=\alpha_{1}\left(t_{0}\right)=\alpha_{2}\left(t_{0}\right) \\
v_{1}=\alpha_{1}^{\prime}\left(t_{0}\right) \\
v_{2}=\alpha_{2}^{\prime}\left(t_{0}\right)
\end{gathered}
$$

En estas condiciones

$$
\begin{aligned}
& d f_{p}\left(v_{1}\right)=\beta_{1}^{\prime}\left(t_{0}\right) \\
& d f_{p}\left(v_{2}\right)=\beta_{2}^{\prime}\left(t_{0}\right)
\end{aligned}
$$

La condición de la definición viene a ser:

$$
\beta_{1}^{\prime}\left(t_{0}\right) \cdot \beta_{2}^{\prime}\left(t_{0}\right)=\lambda(p) \alpha_{1}^{\prime}\left(t_{0}\right) \cdot \alpha_{2}^{\prime}\left(t_{0}\right)
$$

Esta igualdad también debe valer cuando los dos vectores tangentes son el mismo, en consecuencia:

$$
\begin{aligned}
& \beta_{1}^{\prime}\left(t_{0}\right) \cdot \beta_{1}^{\prime}\left(t_{0}\right)=\lambda(p) \alpha_{1}^{\prime}\left(t_{0}\right) \cdot \alpha_{1}^{\prime}\left(t_{0}\right) \\
& \beta_{2}^{\prime}\left(t_{0}\right) \cdot \beta_{2}^{\prime}\left(t_{0}\right)=\lambda(p) \alpha_{2}^{\prime}\left(t_{0}\right) \cdot \alpha_{2}^{\prime}\left(t_{0}\right)
\end{aligned}
$$

Es decir

$$
\begin{aligned}
& \left\|\beta_{1}^{\prime}\left(t_{0}\right)\right\|^{2}=\lambda(p)\left\|\alpha_{1}^{\prime}\left(t_{0}\right)\right\|^{2} \\
& \left\|\beta_{2}^{\prime}\left(t_{0}\right)\right\|^{2}=\lambda(p)\left\|\alpha_{2}^{\prime}\left(t_{0}\right)\right\|^{2}
\end{aligned}
$$


De estas igualdades se sigue que

$$
\frac{\beta_{1}^{\prime}\left(t_{0}\right) \cdot \beta_{2}^{\prime}\left(t_{0}\right)}{\left\|\beta_{1}^{\prime}\left(t_{0}\right)\right\|\left\|\beta_{2}^{\prime}\left(t_{0}\right)\right\|}=\frac{\alpha_{1}^{\prime}\left(t_{0}\right) \cdot \alpha_{2}^{\prime}\left(t_{0}\right)}{\left\|\alpha_{1}^{\prime}\left(t_{0}\right)\right\|\left\|\alpha_{2}^{\prime}\left(t_{0}\right)\right\|}
$$

En los cursos de cálculo vectorial se suele ver la fórmula

$$
\cos \theta=\frac{u \cdot v}{\sqrt{u \cdot u} \sqrt{v \cdot v}}
$$

De lo anterior podemos concluir que $f$ preserva los ángulos.

En la práctica, dado que con dos vectores que apunten en direcciones diferentes podemos generar todos los vectores del plano tangente, no es indispensable comprobar la condición anterior con todos los vectores $\alpha_{i}^{\prime}$ posibles. Es suficiente verificar la condición con dos vectores que apunten en direcciones diferentes en cada uno de los planos tangentes (en general, basta ver que los elementos de una base de cada plano tangente cumplen la condición).

Para un estudio más profundo del concepto de transformaciones conformes remitimos al lector a libros de Geometría Diferencial de Curvas y Superficies (recomendamos [2], [5], [6]).

\section{La proyección central de la esfera sobre cilindro}

Sea $S$ la esfera de radio $R$ centrada en el origen. Está caracterizada por la ecuación

$$
x^{2}+y^{2}+z^{2}=R^{2}
$$

Sea $M$ el cilindro tangente a la esfera $S$ en el ecuador. Está dado por la ecuación

$$
x^{2}+y^{2}=R^{2}
$$

Proyectemos los puntos de $S$ sobre los puntos de $M$, tomando al origen como una fuente de luz. Con la excepción de los polos, a cada punto de la esfera le corresponde un punto en el cilindro. Esto define una función

$$
T: S_{0} \rightarrow M
$$

donde $S_{0}$ denota a la esfera $S$ menos los polos.

La regla de correspondencia se puede formular como sigue:

$$
T(x, y, z)=\left(\frac{R x}{\sqrt{x^{2}+y^{2}}}, \frac{R y}{\sqrt{x^{2}+y^{2}}}, \frac{R z}{\sqrt{x^{2}+y^{2}}}\right)
$$


Para describir con más facilidad el comportamiento de esta función parametrizaremos la esfera con dos ángulos.

$$
\begin{gathered}
x=R \cos \theta \cos \phi \\
y=R \operatorname{sen} \theta \cos \phi \\
z=R \operatorname{sen} \phi
\end{gathered}
$$

Donde:

$R$ es constante, el radio de la esfera.

$\theta$ es el ángulo $A O P^{*}$ donde $A=(R, 0,0), O$ es el origen, y $P^{*}=(x, y, 0)$ es la proyección de $P=(x, y, z)$ sobre el plano $X Y$. A éste ángulo lo llamaremos la longitud. El intervalo en el cual trabajaremos con este ángulo será

$$
0<\theta<2 \pi
$$

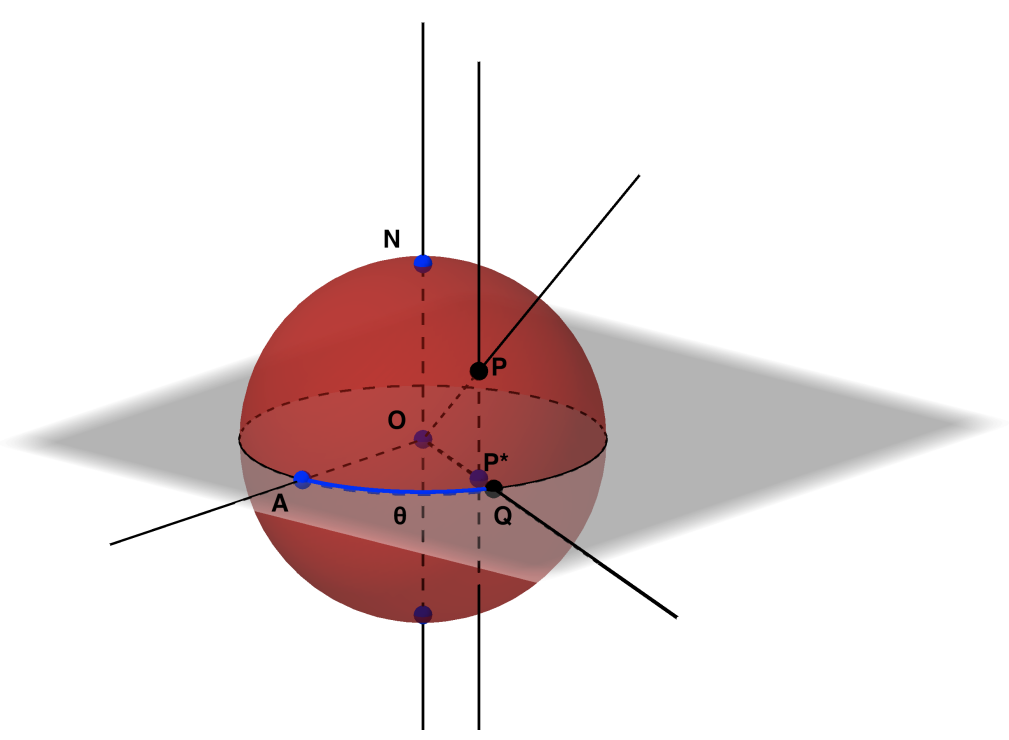

Figura 3: La coordenada longitud

$\phi$ es el ángulo $Q O P$ donde $Q$ es el punto del ecuador cortado por el círculo máximo que pasa por $P$ y por el polo norte $N=(0,0, R)$. A este ángulo lo llamaremos la latitud. El intervalo en el cual trabajaremos con este ángulo será

$$
-\frac{\pi}{2}<\phi<\frac{\pi}{2}
$$

En términos de estas coordenadas, la regla de correspondencia de $T$ queda:

$$
(R \cos \theta \cos \phi, R \operatorname{sen} \theta \cos \phi, R \operatorname{sen} \phi) \mapsto(R \cos \theta, R \operatorname{sen} \theta, R \tan \phi)
$$




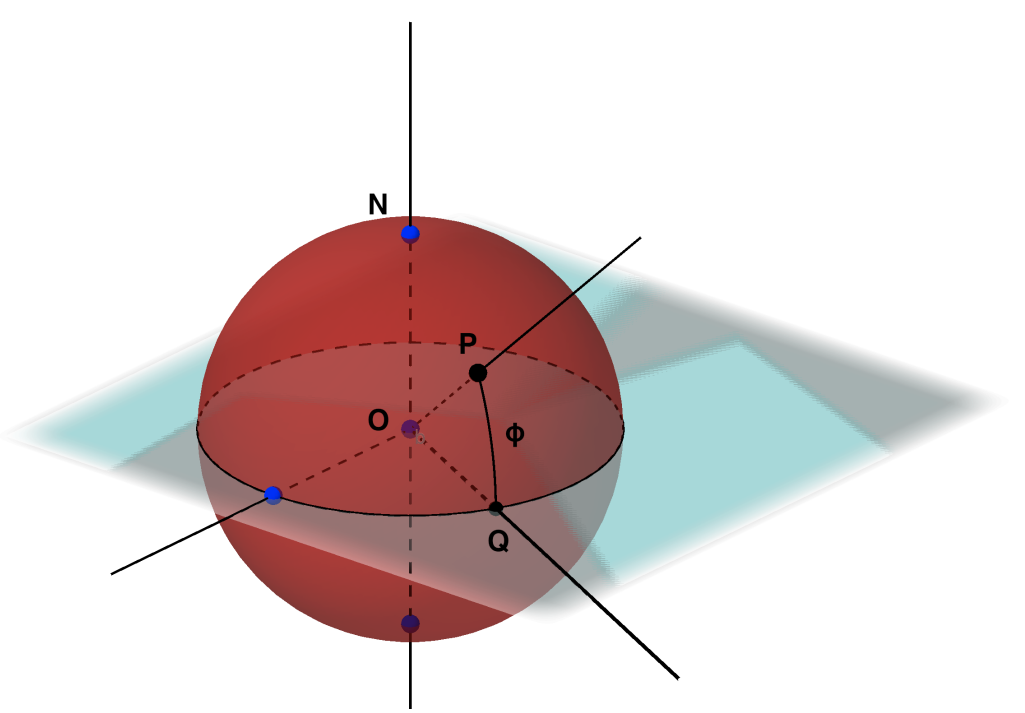

Figura 4: La coordenada latitud.

3.1 La imagen de los paralelos bajo $T$

Un paralelo es un círculo paralelo al ecuador. Está caracterizado por el hecho de que a lo largo de él, la latitud es constante, digamos, $\phi_{0}$.

El paralelo de latitud $\phi=\phi_{0}$ es una circunferencia de radio

\section{$R \cos \phi_{0}$}

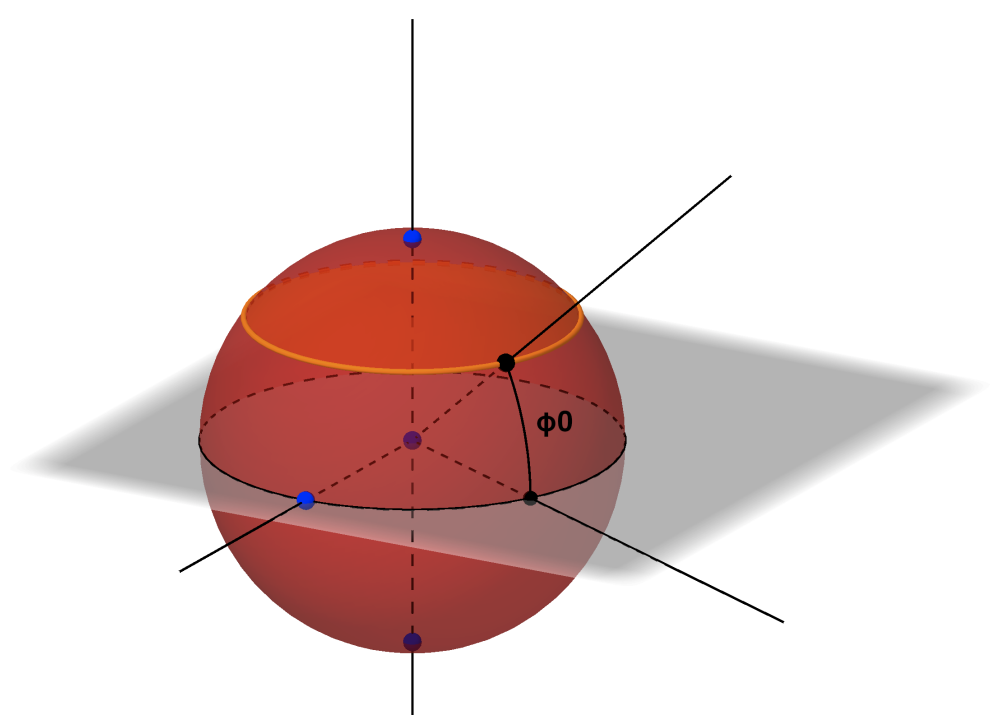

Figura 5: Un paralelo de latitud $\phi_{0}$

Podemos parametrizar el paralelo de latitud $\phi_{0}$ tomando como variable independiente 
$t=\theta$ (podemos pensarlo como la curva que describe un punto que se mueve con velocidad angular unitaria)

$$
\alpha_{1}(t)=\left(R \operatorname{costcos} \phi_{0}, R \operatorname{sent} \cos \phi_{0}, R \operatorname{sen} \phi_{0}\right)
$$

La imagen de esta curva $\alpha_{1}$ es una curva $\beta_{1}$ cuya regla de correspondencia es

$$
\beta_{1}(t)=\left(\text { Rcost, Rsent, Rtan } \phi_{0}\right)
$$

$\beta_{1}$ describe una circunferencia de radio $R$, la cual está en el cilindro.

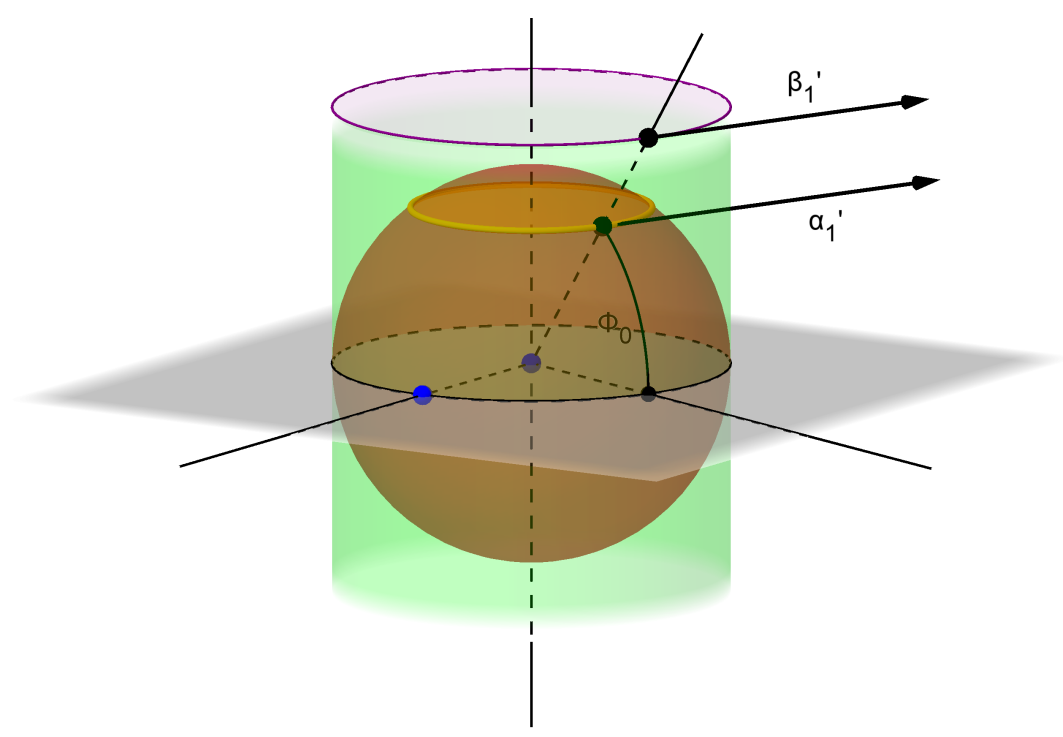

Figura 6: Imagen de un paralelo bajo la proyección central

Calculemos el vector velocidad de la curva $\alpha_{1}$ :

$$
\alpha_{1}^{\prime}(t)=\left(-R \operatorname{sent} \cos \phi_{0}, R \operatorname{cost} \cos \phi_{0}, 0\right)
$$

Nótese que es un vector horizontal.

Calculemos el vector velocidad de la curva $\beta_{1}$ :

$$
\beta_{1}^{\prime}(t)=(-R \operatorname{sent}, \text { Rcost }, 0)
$$

Es otro vector horizontal. Más aún:

$$
\beta_{1}^{\prime}(t)=\sec \phi_{0} \alpha_{1}^{\prime}(t)
$$

Los dos vectores apuntan en la misma dirección, pero tienen magnitudes diferentes: para obtener el vector $\beta_{1}^{\prime}(t)$ basta multiplicar el vector $\alpha_{1}^{\prime}(t)$ por el factor 
$\sec \phi_{0}$

Para saber el factor por el cual se modifican las distancias entre los puntos que pertenecen a una y otra circunferencia, basta calcular la razón de sus longitudes:

$$
\frac{2 \pi R}{2 \pi R \cos \phi_{0}}=\sec \phi_{0}
$$

Esta no es una mera coincidencia, la longitud de una curva parametrizada por el tiempo puede calcularse como la integral de la rapidez en el intervalo de tiempo correspondiente. En el caso que nos ocupa, la rapidez con la que se recorre la curva $\alpha_{1}$ es la constante $R$, mientras que la rapidez con la que se recorre la curva $\beta_{1}$ es la constante $R \sec \phi_{0}$. Pongamos la imagen siguiente: Dos planetas dan una vuelta al sol en el mismo tiempo, pero el segundo viaja el doble de rápido que el primero; podemos concluir que el segundo planeta recorre el doble de distancia que el primero.

\subsection{La imagen de los meridianos bajo $T$}

Un meridiano es una curva que une los dos polos de una esfera, y está contenida en la mitad de un círculo máximo. Está caracterizado por el hecho de que, a lo largo de él, la longitud $\theta$ es constante, digamos $\theta_{0}$. La región delimitada por dos meridianos es como un gajo de esfera.

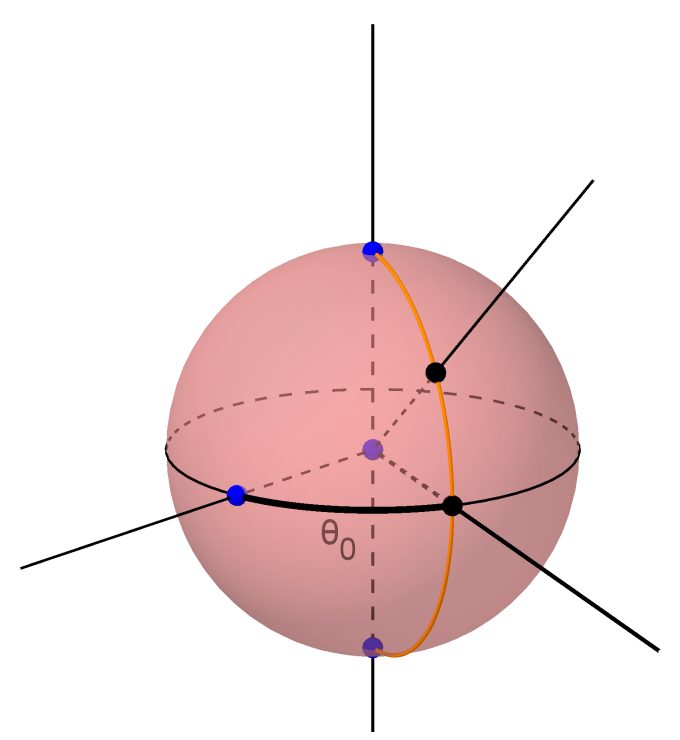

Figura 7: Un meridiano de longitud $\theta_{0}$

Al variar $\phi$ en el intervalo $\left(\frac{-\pi}{2}, \frac{\pi}{2}\right)$, el punto en el cilindro recorre toda la línea vertical determinada por el ángulo $\theta=\theta_{0}$, pues $R \tan \phi$ varía en el intervalo $(-\infty, \infty)$. En este caso las distancias no se deforman de manera homogénea. Las distancias cercanas al ecuador se deforman poco, las distancias cercanas a los polos se alargan muchísimo. Así, el factor de 
alargamiento depende de la latitud del punto.

Podemos parametrizar al meridiano de longitud $\theta_{0}$ tomando como variable independiente $t=\phi$.

$$
\alpha_{2}(t)=\left(R \cos \theta_{0} \operatorname{cost}, R \operatorname{sen} \theta_{0} \cos t, R \operatorname{sen} t\right)
$$

La imagen de esta curva $\alpha_{2}$ es una curva $\beta_{2}$ cuya regla de correspondencia es

$$
\beta_{2}(t)=\left(R \cos \theta_{0}, R \operatorname{sen} \theta_{0}, R \tan t\right)
$$

Calculemos el vector velocidad de la curva $\alpha_{2}$ :

$$
\alpha_{2}^{\prime}(t)=\left(-R \operatorname{sent} \cos \theta_{0},-R \operatorname{sent} \operatorname{sen} \theta_{0}, R \cos t\right)
$$

Calculemos el vector velocidad de la curva $\beta_{2}$ :

$$
\beta_{2}^{\prime}(t)=\left(0,0, \operatorname{Rsec}^{2} t\right)
$$

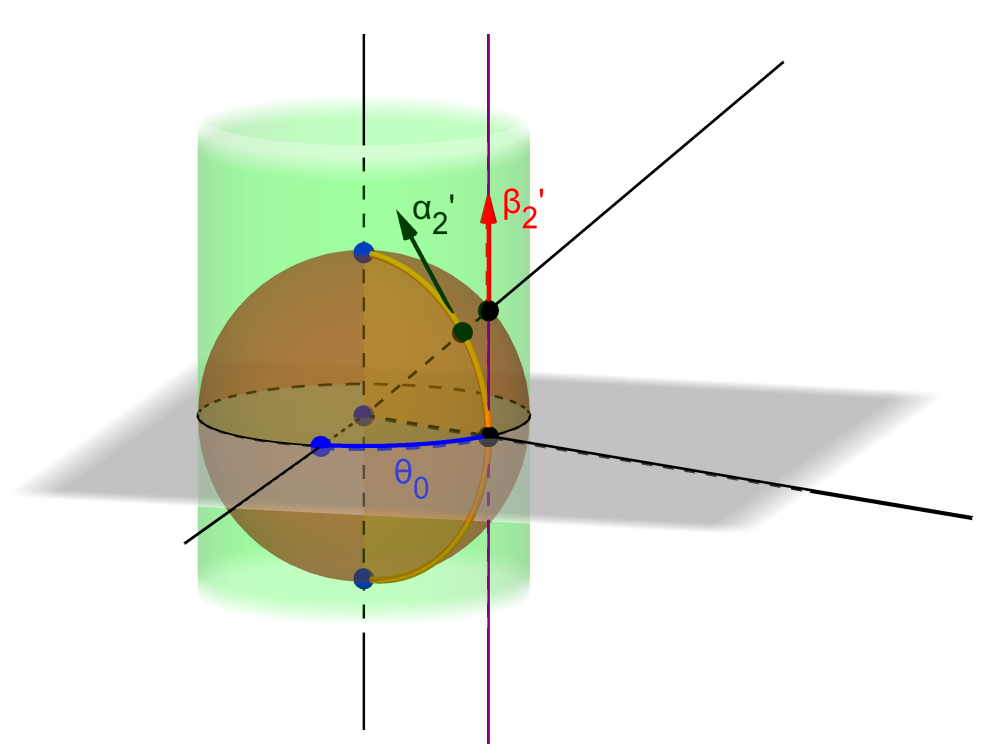

Figura 8: La imagen de un meridiano bajo la proyección central

Esta vez los vectores no apuntan en la misma dirección, sin embargo podemos comparar sus magnitudes en el instante $t_{0}=\phi_{0}$ :

$$
\begin{gathered}
\left\|\alpha_{2}^{\prime}\left(t_{0}\right)\right\|=R \\
\left\|\beta_{2}^{\prime}\left(t_{0}\right)\right\|=R \sec ^{2} t_{0} \\
\frac{\left\|\beta_{2}^{\prime}\left(t_{0}\right)\right\|}{\left\|\alpha_{2}^{\prime}\left(t_{0}\right)\right\|}=\frac{R \sec ^{2} t_{0}}{R}=\sec ^{2} t_{0}
\end{gathered}
$$


El factor por el cual se modifican las distancias cerca del punto $P$ con latitud $\theta_{0}$ y longitud $\phi_{0}$ es $\sec ^{2} \phi_{0}$. Comprobamos que depende de las coordenadas del punto $P$.

\subsection{Esta proyección NO es conforme}

Tomemos un punto $P$ en la esfera perforada $S_{0}$. Supongamos que su latitud es $\phi_{0}$ y su longitud es $\theta_{0}$. Consideremos las curvas $\alpha_{1}$ y $\alpha_{2}$ que parametrizan al paralelo y al meridiano que pasan por el punto $P$, sus vectores velocidad son

$$
\begin{gathered}
\alpha_{1}^{\prime}\left(\theta_{0}\right) \\
\alpha_{2}^{\prime}\left(\phi_{0}\right)
\end{gathered}
$$

Estos vectores son ortogonales, tienen magnitud $R$ y generan a todos los vectores del plano tangente a $S_{0}$ en $P$.

Ahora consideremos las curvas correspondientes en el cilindro $M$, es decir, $\beta_{1}$ y $\beta_{2}$. Dichas curvas son una circunferencia y una línea vertical en el cilindro. Ambas pasan por $Q$, la imagen de $P$ bajo la proyección central. Cuando pasan por $Q$, sus vectores velocidad son

$$
\begin{aligned}
& \beta_{1}^{\prime}\left(\theta_{0}\right) \\
& \beta_{2}^{\prime}\left(\phi_{0}\right)
\end{aligned}
$$

Estos vectores son ortogonales y tienen magnitudes $R \sec \phi_{0}$ y $R \sec ^{2} \phi_{0}$, respectivamente.

La transformación lineal que lleva a los vectores velocidad de las curvas de la esfera que pasan por $P$ en los vectores velocidad de las curvas correspondientes en el cilindro y pasan por $T(P)$, es llamada la diferencial de $T$ en el punto $P$.

Para comprender mejor el comportamiento de la diferencial de $T$, consideremos un ejemplo más sencillo, de una transformación lineal parecida:

Sea

$$
\begin{gathered}
F: \mathbb{R}^{2} \rightarrow \mathbb{R}^{2} \\
(x, y) \mapsto(2 x, 3 y)
\end{gathered}
$$

Los vectores $e_{1}=(1,0)$ y $e_{2}=(0,1)$ son ortogonales y del mismo tamaño. Sus imágenes son los vectores $f_{1}=(2,0)$ y $f_{2}=(0,3)$, que también son ortogonales, pero no son de la misma magnitud. Así, el cuadrado cuyos vértices son el origen, $e_{1}, e_{2}$ y $e_{1}+e_{2}$ va al rectángulo cuyos vértices son el origen, $f_{1}, f_{2}$ y $f_{1}+f_{2}$. En particular, el ángulo que forma la diagonal del cuadrado con los ejes, es diferente del ángulo que forma la diagonal del rectángulo con 


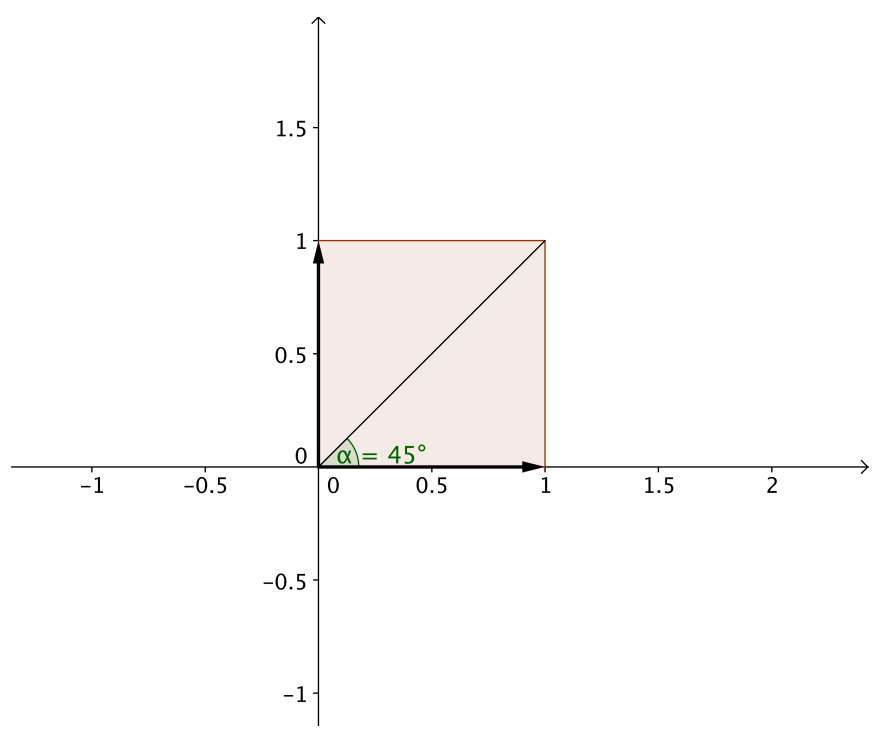

Figura 9: Una base ortonormal en el dominio de $F$

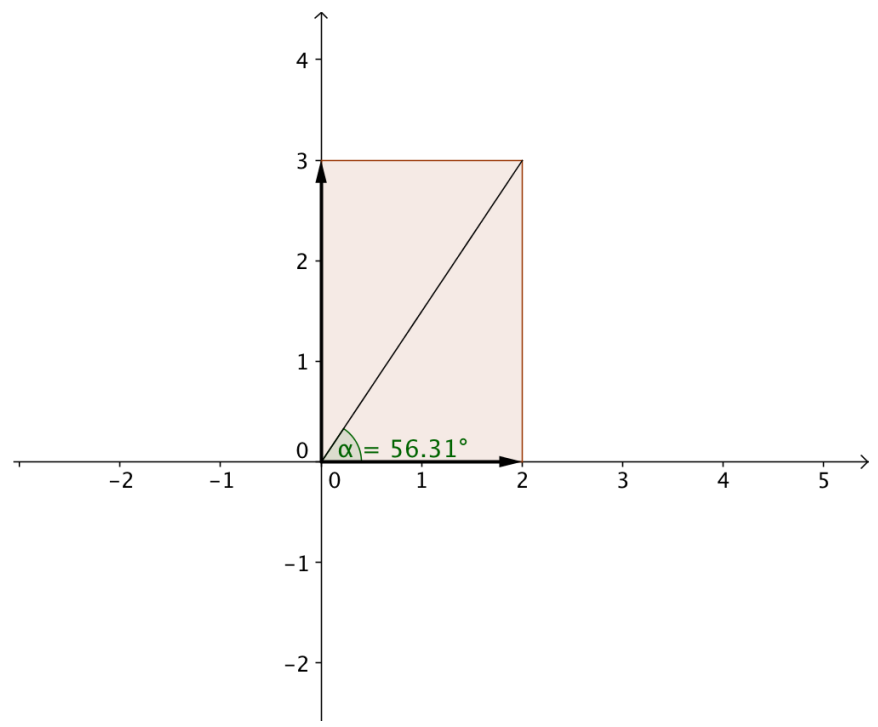

Figura 10: La imagen de dicha base ortonormal

los ejes correspondientes. No preserva los ángulos.

Regresando a la proyección central de la esfera al cilindro tangente, podemos decir que no es conforme porque alarga más las distancias en unas direcciones que en otras. Para que preservara los ángulos tendría que alterar las distancias en todas las direcciones por un mismo factor, y no es el caso.

\section{La proyección desde el eje vertical}

Consideremos ahora 


$$
\begin{gathered}
W: S_{0} \rightarrow M \\
(x, y, z) \mapsto\left(\frac{R x}{\sqrt{x^{2}+y^{2}}}, \frac{R y}{\sqrt{x^{2}+y^{2}}}, z\right)
\end{gathered}
$$

4.1 Imagen de los paralelos bajo $W$

Sea $\alpha_{1}$ igual que en la sección anterior. Sea

$$
\begin{gathered}
\gamma_{1}=W \circ \alpha_{1} \\
\gamma_{1}(t)=\left(R \operatorname{Rcost}, R \operatorname{sen} t, R \operatorname{sen} \phi_{0}\right)
\end{gathered}
$$

Esta curva describe una circunferencia de radio $R$ en el cilindro, cuyo vector velocidad es:

$$
\gamma_{1}^{\prime}(t)=(-R \text { sent, Rcost }, 0)
$$

Este vector se parece mucho al vector velocidad de la curva $\beta_{1}$, pero está anclado en un punto diferente. El factor de amplificación, como antes, es:

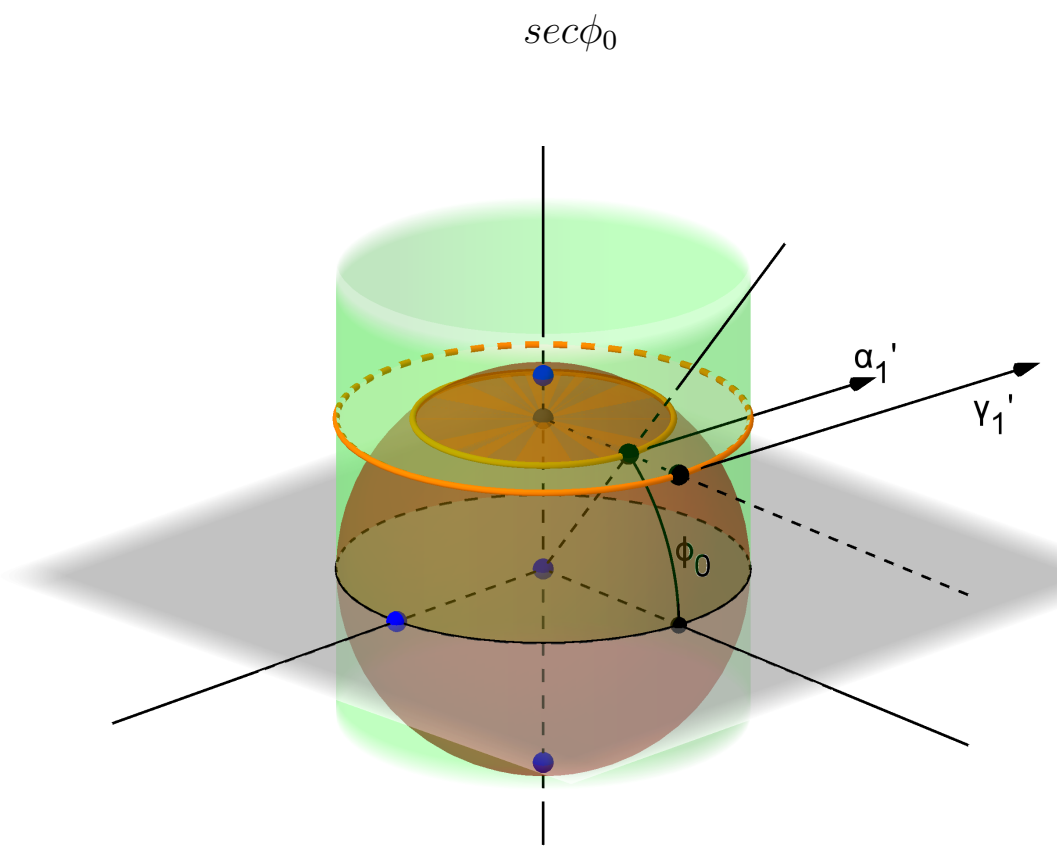

Figura 11: La imagen de un paralelo bajo la proyección desde el eje vertical 
4.2 La imagen de los meridianos bajo $W$

Sea $\alpha_{2}$ igual que antes. Sea

$$
\begin{gathered}
\gamma_{2}=W \circ \alpha_{2} \\
\gamma_{2}(t)=\left(R \cos \theta_{0}, R \operatorname{sen} \theta_{0}, R \operatorname{sen} t\right)
\end{gathered}
$$

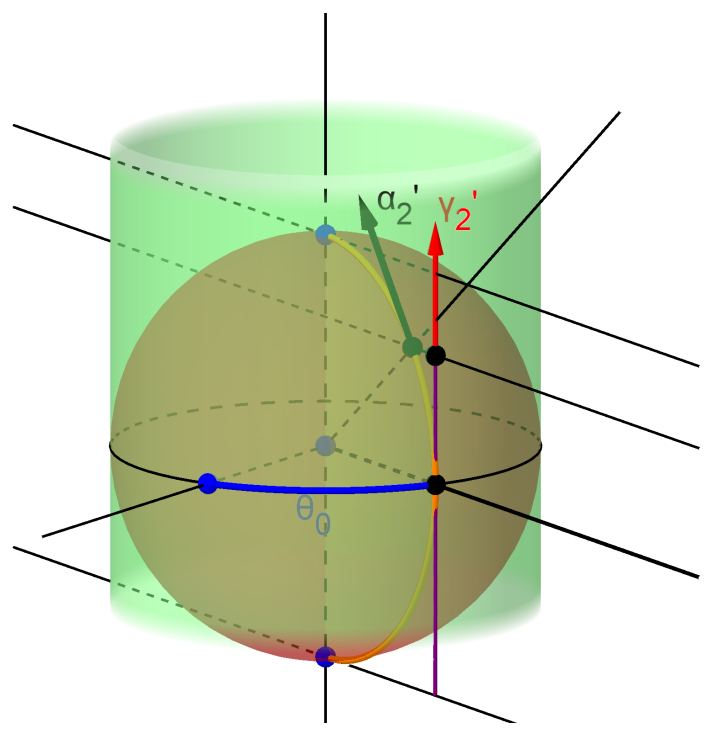

Figura 12: Imagen de un meridiano bajo la proyección desde el eje vertical

El vector velocidad de esta curva es:

$$
\gamma_{2}^{\prime}(t)=(0,0, R \text { cost })
$$

Este vector tiene magnitud

\section{Rcost}

El factor por el cual $W$ modifica las distancias en la dirección vertical se obtiene dividiendo la rapidez de la curva $\gamma_{2}$ entre la rapidez de la curva $\alpha_{2}$

$$
\frac{\left\|\gamma_{2}^{\prime}(t)\right\|}{\left\|\alpha_{2}^{\prime}(t)\right\|}=\frac{R \cos t}{R}=\mathrm{cos} t
$$

Nótese que como $\gamma_{2}^{\prime}(t)$ apunta en la dirección vertical, la traza de la curva es un segmento de recta en el cilindro. 


\subsection{Esta proyección TAMPOCO es conforme}

Tomemos un punto $P$ en la esfera perforada $S_{0}$. Supongamos que $P$ tiene latitud $\phi_{0} \mathrm{y}$ longitud $\theta_{0}$. Consideremos las curvas $\alpha_{1}$ y $\alpha_{2}$ que parametrizan al paralelo y al meridiano que pasan por el punto. Cuando pasan por $P$, sus vectores velocidad son

$$
\begin{aligned}
& \alpha_{1}^{\prime}\left(\theta_{0}\right) \\
& \alpha_{2}^{\prime}\left(\phi_{0}\right)
\end{aligned}
$$

Ambos vectores velocidad son ortogonales y tienen magnitud $R$.

Ahora consideremos las curvas correspondientes en el cilindro $M$, es decir, $\gamma_{1}$ y $\gamma_{2}$. Dichas curvas son una circunferencia y una línea vertical en el cilindro. Ambas pasan por $\bar{P}$, la imagen de $P$ bajo la proyección central. Cuando pasan por $\bar{P}$, sus vectores velocidad son

$$
\begin{aligned}
& \gamma_{1}^{\prime}\left(\theta_{0}\right) \\
& \gamma_{2}^{\prime}\left(\phi_{0}\right)
\end{aligned}
$$

Ambos vectores velocidad son ortogonales y tienen magnitudes $R \sec \phi_{0}$ y $R \cos \phi_{0}$ respectivamente.

Esta transformación no es conforme porque en la dirección vertical acorta los vectores velocidad, mientras que en la dirección horizontal los alarga. Luego, no preserva los ángulos.

\section{La transformación de Mercator}

Ahora plantearemos el problema siguiente:

Construir una transformación $\Psi: S_{0} \rightarrow M$ tal que:

1. Lleve círculos paralelos al ecuador en círculos horizontales.

2. Lleve meridianos en segmentos de recta verticales .

3. Sea conforme.

Las dos proyecciones anteriores cumplen las primeras dos condiciones, pero no la tercera. Propondremos la regla de correspondencia de $\Psi$ de la forma siguiente:

$$
(R \cos \theta \cos \phi, R \operatorname{sen} \theta \cos \phi, R \operatorname{sen} \phi) \mapsto(R \cos \theta, R \operatorname{sen} \theta, f(\phi))
$$

Esto garantiza que los paralelos vayan a dar a círculos horizontales, y que los meridianos sean transformados en segmentos de rectas verticales. Además el problema se reduce a encontrar una función $f$ real de una variable real que permita a $\Psi$ satisfacer la tercera condición. 
5.1 La imagen de los paralelos bajo $\Psi$

Tomemos un paralelo parametrizado por

$$
\alpha_{1}(t)=\left(R \operatorname{costcos} \phi_{0}, R \operatorname{sent} \cos \phi_{0}, R \operatorname{sen} \phi_{0}\right)
$$

Tendremos, como antes, que

$$
\alpha_{1}^{\prime}(t)=\left(-R \operatorname{sent} \cos \phi_{0}, R \operatorname{costcos} \phi_{0}, 0\right)
$$

Sea $\delta_{1}=\Psi \circ \alpha_{1}$. Entonces

$$
\delta_{1}(t)=\left(\text { Rcost }, \text { Rsent }, f\left(\phi_{0}\right)\right)
$$

y se tiene que

$$
\delta_{1}^{\prime}(t)=(-R \operatorname{sen} t, R \cos t, 0)
$$

5.2 Imagen de los meridianos bajo $\Psi$

Sea $\alpha_{2}(t)=\left(R \cos \theta_{0} \operatorname{cost}, R \operatorname{sen} \theta_{0} \cos t, R \operatorname{sen} t\right)$, de donde

$$
\alpha_{2}^{\prime}(t)=\left(-R \cos \theta_{0} \operatorname{sen} t,-R \operatorname{sen} \theta_{0} \operatorname{sen} t, R \cos t\right) .
$$

Sea $\delta_{2}=\Psi \circ \alpha_{2}$, entonces

$$
\delta_{2}(t)=\left(R \cos \theta_{0}, R \operatorname{sen} \theta_{0}, f(t)\right) .
$$

Derivando obtenemos

$$
\delta_{2}^{\prime}(t)=\left(0,0, f^{\prime}(t)\right)
$$

\subsection{La condición necesaria para que $\Psi$ sea conforme}

Pensemos por un momento qué falló con las primeras dos proyecciones que analizamos. La proyección central alarga los vectores velocidad verticales más que los vectores horizontales. La proyección desde el eje vertical acorta los vectores verticales mientras alarga los vectores horizontales. Ambas tienen un efecto diferenciado en las longitudes de los vectores velocidad de las curvas consideradas.

Para que $\Psi$ preserve los ángulos se necesita que modifique por el mismo factor los vectores velocidad en todas las direcciones. Intuitivamente, esto significa que localmente se comporte como una homotecia que amplifique las distancias por el mismo factor en todas 
las direcciones; ésta es la condición que permitirá que $\Psi$ preserve los ángulos.

En la dirección horizontal (en el paralelo de latitud $\phi_{0}$ ) el factor por el cual se modifica la rapidez de los vectores velocidad es

$$
\sec \phi_{0}
$$

En la dirección vertical (en el meridiano de longitud $\theta_{0}$ ) el factor por el cual se modifica la rapidez de los vectores velocidad es $\frac{f^{\prime}\left(\phi_{0}\right)}{R}$.

La condición de que en ambas direcciones el factor de amplificación sea el mismo se reduce a

$$
f^{\prime}\left(\phi_{0}\right)=R \sec \phi_{0}
$$

Si esto debe cumplirse en todos los puntos

$$
f(\phi)-f(0)=\int_{0}^{\phi} R \sec t d t
$$

Partiendo de que en el ecuador se debe cumplir $f(0)=0$ tendremos que

$$
f(\phi)=R \int_{0}^{\phi} \operatorname{sect} d t=R \ln (\sec \phi+\tan \phi)
$$

Entonces, la transformación de Mercator es:

$$
(R \cos \theta \cos \phi, R \operatorname{sen} \theta \cos \phi, R \operatorname{sen} \phi) \mapsto(R \cos \theta, R \operatorname{sen} \theta, R \ln (\sec \phi+\tan \phi))
$$

\subsection{Interpretando un mapamundi}

Para finalizar este artículo consideraremos la cuestión de interpretar un mapamundi elaborado con la transformación de Mercator. Tomemos en cuenta que las rectas en el mapa no corresponden a rectas en la tierra. Para ser más precisos:

1. En el mapamundi, una línea horizontal representa una circunferencia paralela al ecuador (un paralelo).

2. En el mapamundi, una línea vertical representa una semicircunferencia que une los polos (un meridiano).

3. Las otras líneas rectas representan un cierto tipo de curvas llamadas loxodromos. Son como espirales que van enrollándose en torno a los polos. 
4. Un defecto que tiene la proyección de Mercator: el camino más corto entre dos puntos en la superficie terrestre (un círculo máximo) no queda representado por un segmento de recta en el mapa.

5. Una virtud que tiene la proyección de Mercator: en el mapa podemos saber en qué dirección navegar para llegar al destino que hayamos elegido.

\section{Referencias}

[1] Carslaw, H.S., The Story of Mercator's Map: A Chapter in the History if Mathematics, The Mathematical Gazette, Vol 12, No. 168, pp. 1-7, (1924).

[2] Do Carmo, Manfredo. Geometría Diferencial de Curvas y Superficies, 500 páginas, Alianza, Madrid, (1990).

[3] Guillemin, V., Pollack, A. Topología Diferencial. Colección papirhos. Instituto de Matemáticas, UNAM, (2015).

[4] Havernals, N. and Roscoe, M., The history of mathematics as a pedagogical tool: Teaching the integral of the secant via Mercator's projection, The Mathematics Entusiast, vol. 7, No. 2, pp. 339-368 (2010).

[5] O’Neill, Barret. Elementary Differential Geometry, Academic, Boston, (2006).

[6] Palmas Velasco, Oscar; Reyes Victoria, J.G. Curso de geometrí diferencial. Las Prensas de Ciencias, UNAM, (2005).

[7] Rickey, F. and Tuchinsky, P.M.. An Application of Geography to Mathematics: History of the Integral of the Secant, Mathematics Magazine, Vol. 53, No. 3, pp. 162-166 (1980). 